\title{
Cuidado de enfermagem forense ao idoso em situações de violência: revisão de escopo
}

Forensic nursing care for older adults in situations of violence: a scoping review Cuidados de enfermería forense a adultos mayores en situación de violencia: revisión de alcance

Jiovana de Souza Santos ${ }^{1}$ (D https://orcid.org/0000-0001-6056-8800

Rafael da Costa Santos ${ }^{1}$ iD https://orcid.org/0000-0001-8293-340X

Gleicy Karine Nascimento de Araújo-Monteiro' ${ }^{1}$ id htps://orcid.org/0000-0002-4395-6518

Renata Clemente dos Santos ${ }^{1}$ id https://orcid.org/0000-0003-2916-6832

Gabriela Maria Cavalcanti Costa ${ }^{1}$ ic https://orcid.org/0000-0003-4466-6886

Raúl Fernando Guerrero-Castañeda² ic https://orcid.org/0000-0003-3996-5208

Rafaella Queiroga Souto ${ }^{1}$ io https://orcid.org/0000-0002-7368-8497 Como citar:
Santos JS, Santos RC, Araújo-Monteiro GK, Santos RC, Costa GM, Guerrero-Castañeda RF, et al. Cuidado de enfermagem forense ao idoso em situações de violência: revisão de escopo. Acta Paul Enferm. 2021;34:AAPE002425.

DOI http://dx.doi.org/10.37689/actaape/2021AR02425

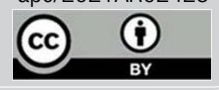

$$
\text { Descritores }
$$

Cuidados de enfermagem; Enfermagem forense; Idoso; Maus-tratos ao idoso; Violência

Keywords

Aged; Elder abuse; Forensic nursing; Nursing care; Violence

Descriptores Anciano; Atención de enfermeira; Enfermería forense; Maltrato al anciano; Violencia

$$
\begin{array}{r}
\text { Submetido } \\
29 \text { de Agosto de } 2020 \\
\text { Aceito } \\
16 \text { de Março de } 2021
\end{array}
$$

Autor correspondente

Rafaella Queiroga Souto

E-mail: rafaellaqueiroga7@gmail.com

\section{Resumo}

Objetivo: Descrever o cuidado da enfermagem forense ao idoso em situação de violência.

Métodos: Trata-se de uma scoping review com base nas recomendações do Joanna Briggs Institute. As buscas ocorreram em 15 bases de dados, tendo como inclusão estudos publicados entre os anos de 1990 a 2019, nas línguas: inglesa, francesa, espanhola e portuguesa. Para seleção dos estudos foi utilizado 0 diagrama de fluxo Preferred Reporting Items for Systematic reviews and Meta-Analyses extension for Scoping Reviews. Foram encontrados 17.378 estudos, destes, 19 artigos foram elegíveis para a revisão.

Resultados: 0 cuidado da enfermagem ao idoso em situações de violência é dinâmico e varia entre os continentes. Os enfermeiros investigam o caso por meio de avaliação clínica, denunciam as autoridades, registram, notificam e acionam a equipe multidisciplinar.

Conclusão: Lançando mão de estratégias diversificadas, os enfermeiros atuam com objetivo de solucionar 0 problema da violência contra o idoso, ainda que, encontrem dificuldades.

\section{Abstract}

Objective: To describe forensic nursing care for older adults in situations of violence.

Methods: This is a scoping review based on the Joanna Briggs Institute recommendations. The searches took place in 15 databases, including studies published between 1990 and 2019 in English, French, Spanish, and Brazilian Portuguese. For study selection, the flow diagram Preferred Reporting Items for Systematic reviews and Meta-Analyzes extension for Scoping Reviews was used; 17,378 studies were found, of which 19 articles were eligible for review.

Results: Nursing care for older adults in situations of violence is dynamic and varies across continents. Nurses investigate the case through clinical assessment, denounce the authorities, register, notify and activate the multidisciplinary team.

Conclusion: Using diversified strategies, nurses work with the objective of solving the problem of elder abuse, even if they encounter difficulties.

\section{Resumen}

Objetivo: Describir los cuidados de enfermería forense a adultos mayores en situación de violencia.

Métodos: Se trata de un scoping review basado en las recomendaciones del Joanna Briggs Institute. Las búsquedas se llevaron a cabo en 15 bases de datos e incluyeron estudios publicados entre los años 1990 y 2019, en idioma inglés, francés, español y portugués. Para la selección de los estudios se utilizó el diagrama 
de flujo Preferred Reporting Items for Systematic reviews and Meta-Analyses extension for Scoping Reviews. Se encontraron 17.378 estudios, de los cuales 19 fueron elegibles para la revisión.

Resultados: Los cuidados de enfermería a los adultos mayores en situación de violencia son dinámicos y varían entre continentes. Los enfermeros investigan el caso mediante evaluación clínica, denuncian ante las autoridades, registran, notifican y llaman al equipo multidisciplinario.

Conclusión: Utilizando estrategias diversificadas, los enfermeros actúan con el objetivo de solucionar el problema de la violencia contra los adultos mayores, aunque enfrenten dificultades.

\section{Introdução}

A enfermagem forense (EF) é uma área recentemente consolidada no Brasil. Trata-se da aplicação da ciência da enfermagem aos aspectos forenses do cuidado à saúde. ${ }^{(1)}$ Em outros países, a EF é bem mais desenvolvida quando comparada ao contexto brasileiro, a exemplo dos EUA onde foi fundada a International Association of Forensic Nursing (IAFN), em 1992, por enfermeiros que atuavam como examinadores de abuso sexual e, em 1995, a American Nurses Association (ANA) reconheceu a EF como uma especialidade. ${ }^{(2)}$ No Brasil, esse reconhecimento só ocorreu no ano de 2011, porém apenas em 2017 o Conselho Federal de Enfermagem emite uma resolução com as áreas de atuação desse profissional. ${ }^{(1)}$

A EF tem um papel social relevante e traz novas possibilidades para a prática de enfermagem, possibilitando o desenvolvimento de habilidades adicionais que permitam que o enfermeiro intervenha em situaçóes de violência no âmbito do Sistema Único de Saúde (SUS) e no contexto da privação de liberdade. ${ }^{(3)}$

O Enfermeiro Forense pode atuar em casos de violência em diferentes áreas e ciclos de vida. Dentre as áreas de atuação pode-se citar os casos de maus tratos, trauma, investigação de morte, consultoria, violência sexual, situações carcerárias, psiquiátricas, preservação de vestígios e desastres de massa. ${ }^{(2)}$

Sua área de atuação é ampla e se torna indispensável para a prestação de cuidados às vítimas de violência uma vez que, o profissional enfermeiro, muitas vezes, é o primeiro a atender a pessoa vítima de violência no serviço de saúde. ${ }^{(4)}$ Além disso, a violência é um problema crescente ao longo dos anos e envolve questôes sociais, econômicas, políticas e culturais. ${ }^{(5)}$ Por se tratar de um fenômeno sistêmico, a violência tem impacto direto nos sistemas de saúde, segurança e previdenciário, interferindo de forma negativa na qualidade de vida das pessoas, e por isto, o desenvolvimento da EF no país pode contribuir para melhoria da qualidade de vida das vítimas, além de prevenir situações de violência. ${ }^{(6)}$

A EF pode auxiliar também na promoção da cultura de paz e na prevenção de casos de violência, por meio do planejamento de açóes educativas, direcionadas aos profissionais, mas também a comunidade em geral, que pode incluir vítimas e agressores, com objetivo de disseminar o conhecimento sobre a identificação de sinais e sintomas de violência, fortalecimento de vínculos familiares, estabelecimento de relacionamentos saudáveis, fortalecimento da intergeracionalidade, disponibilização de informaçóes sobre a rede de atendimento a vítimas de violência com respectivos contatos telefônicos e sites, mecanismos de denúncia, assim como o atendimento adequado às vítimas e condução dos casos com os devidos encaminhamentos.

Outro aspecto importante para apoiar a ascensão da prática da EF no Brasil é o fato de que a população do país tem envelhecido de forma acelerada; concomitantemente a isto, ocorre o aumento da violência na faixa etária de pessoas com 60 anos ou mais, e em algum momento estas pessoas precisarão ser atendidas em algum serviço de saúde. ${ }^{(3)}$ A Organização Mundial de Saúde (OMS), destaca que o número de idosos que sofre algum tipo de violência é preocupante, sendo um a cada seis idosos em todo o mundo, além disso, a violência contra a pessoa idosa (VCPI) é pouco diagnosticada e notificada. ${ }^{(7)}$ Isso pode estar relacionado a falta de capacitação profissional, ao medo e a complexidade em identificar os casos de VCPI. ${ }^{(8)}$

$\mathrm{Na}$ suspeita ou confirmação de violência, o caso deverá ser obrigatoriamente notificado, devendo o profissional de saúde encaminhar os casos ao Ministério Público, autoridade policial, ou Conselho do Idoso. ${ }^{(9)} \mathrm{A}$ identificação da VCPI tem caráter emergencial, ao considerar que, cada vez que o idoso frequenta o serviço de saúde, pode ser a úni- 
ca chance de identificar uma situação de violência. (10) A violência resulta em problemas físicos, psicológicos, financeiros, sócias, incapacidade funcional e até mesmo a morte. ${ }^{(11)}$

Assim, o enfermeiro se torna um intermediador na identificação da VCPI por ser de difícil detecção, necessitando de um olhar acurado para perceber os sinais de alerta. Estes, muitas vezes, camuflados em acidentes ou dores recorrentes. ${ }^{(5)}$ Autores ressaltam que, para identificar situaçóes de violência, deve-se lançar mão de estratégias para a sua detecção, utilizando instrumentos validados, escuta qualificada nas consultas de enfermagem, e atuação na disseminação de informaçóes sobre a violência. ${ }^{(6)}$

O comprometimento do profissional enfermeiro que faz uso do conhecimento da EF para detecção de violência pode contribuir para o cuidado humanizado na medida em que as intervençóes podem romper o ciclo de violência. Essas intervençôes devem ser individualizadas e pautadas em evidências científicas, de forma planejada, conforme legislaçáo vigente, políticas públicas de saúde e instrumentos de enfermagem básicos no intuito de mitigar os agravos perpetrados às vítimas de violência. ${ }^{(5)}$

Não foi identificado nenhuma revisão semelhante em andamento no PROSPERO, no MEDLINE, no banco de dados Cochrane Systematic Reviews e no Systematic Reviews Database and Implementation Reports do Joanna Briggs Institute, o presente estudo se justifica e objetiva descrever o cuidado da enfermagem forense ao idoso em situação de violência.

\section{Métodos}

Trata-se de um estudo de Scoping Review, guiado pelas diretrizes metodológicas propostas pelo Joanna Briggs Institute (JBI) e, atendendo a iniciativa PRISMA-ScR. ${ }^{(12)}$ A revisão de escopo (scoping review ou scoping study) objetiva o mapeamento dos principais conceitos utilizados em determinada área do conhecimento, e a identificação de lacunas nas evidências existentes. ${ }^{(13)}$

Para construção da questão norteadora utilizou-se a estratégia PCC, sendo "P" para População enfermeiros, "C" Conceito - o cuidado ao idoso, e
"C" Contexto - violência, com base nessas informaçôes foi estabelecida a seguinte pergunta: quais os cuidados de EF ao idoso em situações de violência? Este cuidado foi avaliado pela descrição da prática apontada nos estudos e realizada por estes profissionais, verificando se estão relacionadas as práticas consideradas forenses.

Foram elegíveis estudos que abordaram o cuidado a pessoa idosa em situaçáo de violência realizado por enfermeiros de modo geral, com o cuidado de selecionar aquelas açóes que poderiam/deveriam ser realizadas com maior esmero e competência técnica pelo enfermeiro forense, nos idiomas em inglês, espanhol, português e francês, disponíveis na íntegra entre os anos de 1990 a 2019, esse intervalo de tempo se justifica por se tratar do período de fundação da International Association of Forensic Nursing em 1990. Ressalta-se que, foram considerados estudos que incluíram enfermeiros na amostra, ainda que, também tenha sido incluído outros profissionais no estudo. No entanto, apenas os dados referentes as respostas dos enfermeiros foram caracterizados como resultados para esta revisão.

O levantamento dos estudos na literatura foi feito entre fevereiro e abril de 2020, por dois pesquisadores de forma independente. Inicialmente foi realizada uma pesquisa usando as palavras-chave $\mathrm{el}$ derly, nursing care e violence em duas bases de dados: MEDLINE via PubMed e CINAHL via EBSCO. Neste momento, foram analisadas as palavras componentes de títulos, resumos e descritores. Os estudos que atendiam ao objetivo foram lidos na íntegra e tiveram suas referências analisadas. Através dessa primeira investigação foram elaboradas as estratégias de busca implementadas para rastreio dos documentos que atendessem ao objetivo da revisão.

Utilizou-se as seguintes palavras-chave e descritores combinados pelos operadores boolenos AND e OR: (("Nurses" OR "Forensic nursing" OR "Nurse examiner" OR "Forensic examination" OR "Nurse's role" OR "Nursing Role" OR "Care nurse" OR "Nursing care" OR "Forensic nurse" OR "Investigating forensic nursing" OR "Sexual assault nurse examiners") AND ("Elder" OR "Aged” OR "Elderly" OR “Older" OR "Older adults") AND ("Abuse" OR "Violence" OR "Sexual violence" OR 
"Mistreatment" OR "Sexual assault" OR "Abused" OR "Traumatic injuries" OR "Victims of violence" OR "Domestic violence" OR "Neglect" OR “Crime victims" OR "Strangulation" OR "Exploitation" OR "Interpersonal violence" OR "Intimidation" OR "Financial abuse")).

A estratégia supracitada foi utilizada nas seguintes bases de dados: MEDLINE via PubMed, CINAHL via EBSCO e plataforma Web of Science. Para as demais bases de dados e plataformas, foi aplicada as seguintes táticas de busca: Nurse Care AND Elderly AND abuse e (("Nurse" OR "Forensic nursing" OR "Nursing care") AND ("Elder" OR "Aged" OR "Elderly") AND ("Abuse” OR "violence")). Em bases que necessitavam alguma adequação, foram utilizados também os Descritores em Ciências da Saúde (DeCs), principalmente em bases da língua portuguesa, implementando a seguinte estratégia: cuidado de enfermagem AND idoso AND violência.

Foram incluídos nesta scoping review as seguintes bases de dados: MEDLINE via PubMed, CINAHL via EBSCO, LILACS, Embase, Scopus, PsycINFO, Banco de Dados JBI de Revisóes Sistemáticas e Relatórios de Implementação, das plataformas Cochrane (ensaios controlados e revisões sistemáticas), e Web of Science. A pesquisa na literatura cinzenta incluiu: MedNar, Portal de Teses e Dissertaçôes da CAPES, DART Portal Europeu de E-Teses, Theses Canadá, Google Acadêmico e Centro de Estudos e Pesquisas em Enfermagem - CEPEN.

Nas bases de dados inicialmente identificou-se 17.378 estudos, após a leitura de títulos, 140 foram selecionados por sugerir discutir o cuidado do enfermeiro em casos de VCPI, e seguiram para a etapa de avaliação exaustiva dos resumos, 29 realmente abordaram a atuação do enfermeiro a pessoa idosa em situação de violência, e passaram para etapa de leitura do texto completo, desses 18 atendiam ao objetivo da revisão. Após a análise das referências um estudo foi incluído, totalizando 19 documentos, conforme apresentado na adaptação do Fluxograma PRISMA-ScR ${ }^{(12)}$ (Figura 1).

Os dados foram extraídos utilizando um instrumento de extraçáo de dados desenvolvido pelos revisores, que incluiu detalhes específicos sobre o cuidado do enfermeiro ao idoso em situaçôes de violência, dificuldades relatadas pelos enfermeiros, além dos metadados (autoria, abordagem metodológica, nível de atenção à saúde, país, ano de publicação, base de dados e tipo de publicação).

O cuidado realizado pelos enfermeiros foi categorizado em medidas de prevenção, recursos utilizados, intervençóes, encaminhamentos, denúncias e as dificuldades relatadas pelos enfermeiros para prestar o cuidado ao idoso em situação de violência. Estas, foram categorizadas em problemas organizacionais, capacitação profissional e, questóes pessoais.

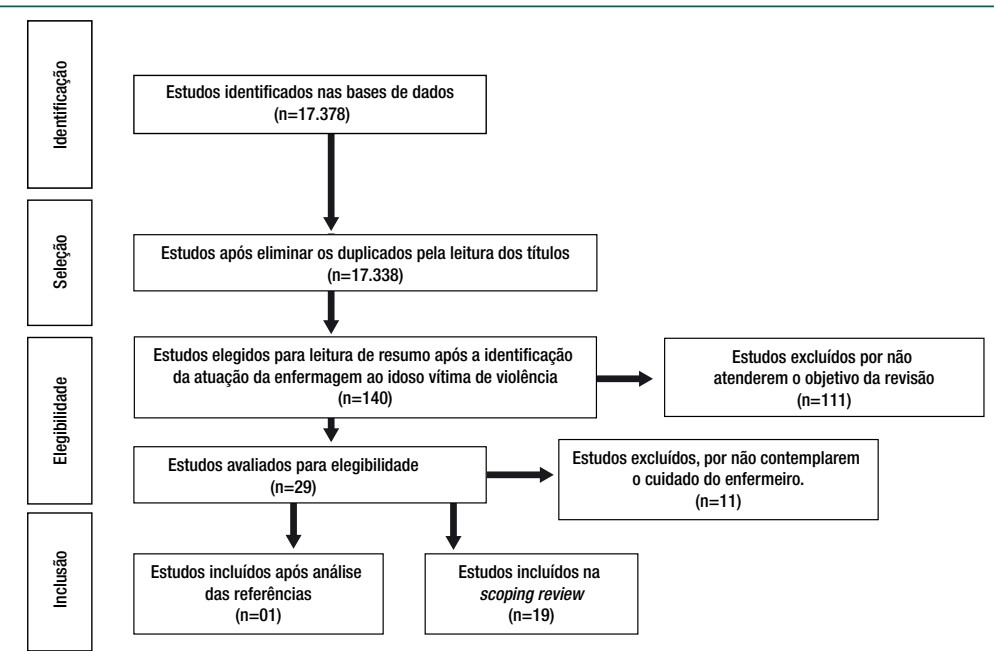

Figura 1. Fluxograma do processo de seleção dos estudos adaptado do PRISMA 


\section{Resultados}

\section{Características dos estudos}

O tipo de publicação foi predominantemente de artigos na língua inglesa $(73,7 \%)$. Cinco artigos estavam na língua portuguesa. Destaca-se a prevalência de estudos com abordagem qualitativa $(73,7 \%)$, seguida de estudos quantitativos $(26,3 \%)$.

Em relação ao país de origem da publicação, a maioria foi no Brasil (26,3\%), seguido da Austrália e Estados Unidos da América (EUA), com (15,8\%) respectivamente, Israel com $(10,5 \%)$ das publicações, Irlanda, Coreia do Sul, Irã, Suíça, Japão, Canadá com $(5,2 \%)$ das publicaçóes respectivamente. No que concerne ao nível de atenção à saúde em que os enfermeiros atuam, predominou Atenção Primária $(36,8 \%)$ seguido da atenção hospitalar e domiciliar $(31,6 \%)$ respectivamente. O tipo de violência mais abordada nos estudos foi a violência geral $(84,2 \%)$. Os metadados dos estudos estáo apresentados no quadro 1 .

\section{Quadro 1. Caracterização das publicações}

\begin{tabular}{|c|c|c|c|c|c|c|c|}
\hline Título & Ano & $\begin{array}{l}\text { Tipo de violência } \\
\text { abordada }\end{array}$ & $\begin{array}{l}\text { Nível de atenção à } \\
\text { saúde }\end{array}$ & $\begin{array}{l}\text { Abordagem } \\
\text { metodológica }\end{array}$ & País do estudo & Base de dados & $\begin{array}{l}\text { Tipo de } \\
\text { publicação }\end{array}$ \\
\hline $\begin{array}{l}\text { Safeguarding staff's experience of cases of } \\
\text { financial abuse }^{(8)}\end{array}$ & 2018 & Violência financeira & Domiciliar & Qualitativa & Irlânda & CINAHL & Artigo \\
\hline $\begin{array}{l}\text { Working at the frontline in cases of elder abuse: } \\
\text { 'It keeps me awake at night }{ }^{(14)}\end{array}$ & 2014 & Violência no geral & Domiciliar & Qualitativa & Austrália & CINAHL & Artigo \\
\hline $\begin{array}{l}\text { Violência contra o idoso: as concepções dos } \\
\text { profissionais de enfermagem sobre detecção e } \\
\text { prevenção }^{(15)}\end{array}$ & 2018 & Violência no geral & Atenção Primária & Qualitativa & Brasil & CINAHL & Artigo \\
\hline $\begin{array}{l}\text { Atuação do enfermeiro perante a violência } \\
\text { doméstica sofrida pelo idoso }{ }^{(16)}\end{array}$ & 2015 & Violência doméstica & Atenção Primária & Quantitativa & Brasil & Google Acadêmico & Artigo \\
\hline $\begin{array}{l}\text { Cuidados de Enfermagem para detecção de } \\
\text { violência contra idosos }{ }^{(17)}\end{array}$ & 2019 & Violência no geral & Hospitalar & Qualitativa & Brasil & Google Acadêmico & Artigo \\
\hline $\begin{array}{l}\text { Attitudes and knowledge of medical and nursing } \\
\text { staff toward elder abuse } \\
\text { (18) }\end{array}$ & 2010 & Violência no geral & Hospitalar & Quantitativa & Israel & Embase & Artigo \\
\hline Elder Abuse. The nurse's perspective ${ }^{(19)}$ & 2005 & Violência no geral & Domiciliar & Qualitativa & $E^{\prime} A^{*}$ & Embase & Artigo \\
\hline $\begin{array}{l}\text { Nurses' encounters with older adults engaged } \\
\text { in self-neglectful behaviors in the community: a } \\
\text { qualitative study }{ }^{(20)}\end{array}$ & 2018 & Autonegligência & Atenção Primária & Qualitativa & Israel & Embase & Artigo \\
\hline $\begin{array}{l}\text { Factors related to Korean nurses' Willingness to } \\
\text { report suspected elder abuse }{ }^{(21)}\end{array}$ & 2012 & Violência no geral & Hospitalar & Quantitativa & Coreia do Sul & Embase & Artigo \\
\hline $\begin{array}{l}\text { Nurses' clinical assessments of older clients who } \\
\text { are suspected victims of abuse: an exploratory } \\
\text { study in community care in Norway }{ }^{(22)}\end{array}$ & 2010 & Violência no geral & Domiciliar & Qualitativa & Noruega & $\begin{array}{l}\text { North Grey } \\
\text { Literature Collection } \\
\text { (MedNar) }\end{array}$ & Artigo \\
\hline $\begin{array}{l}\text { Challenges in handling elder abuse in community } \\
\text { care. An exploratory study among nurses and } \\
\text { care coordinators in Norway and Australia }{ }^{(23)}\end{array}$ & 2011 & Violência no geral & Domiciliar & Qualitativa & Noruega e Austrália & $\begin{array}{l}\text { North Grey } \\
\text { Literature Collection }\end{array}$ & Artigo \\
\hline $\begin{array}{l}\text { Identifying and handling abused older clients in } \\
\text { community care: the } \\
\text { perspectives of nurse managers }{ }^{(24)}\end{array}$ & 2011 & Violência no geral & Domiciliar & Qualitativa & Noruega & $\begin{array}{l}\text { North Grey } \\
\text { Literature Collection }\end{array}$ & Artigo \\
\hline $\begin{array}{l}\text { Critical care nurses' perspectives on elder } \\
\text { abuse }^{(25)}\end{array}$ & 2012 & Violência no geral & Hospitalar & Qualitativa & $E^{\prime} A^{*}$ & $\begin{array}{l}\text { North Grey } \\
\text { Literature Collection }\end{array}$ & Artigo \\
\hline $\begin{array}{l}\text { The relationship between nurses' recognition } \\
\text { regarding elder abuse and their } \\
\text { attitudes and performance in dealing with elder } \\
\text { abuse induced by iranian } \\
\text { family caregivers }\end{array}$ & 2019 & Violência no geral & Hospitalar & Quantitativa & Irã & Scopus & Artigo \\
\hline $\begin{array}{l}\text { Aspectos relacionados à violência contra o idoso: } \\
\text { concepção do enfermeiro da estratégia saúde } \\
\text { da família }{ }^{(27)}\end{array}$ & 2019 & Violência no geral & Atenção Primária & Qualitativa & Brasil & LILACS & Artigo \\
\hline $\begin{array}{l}\text { Enfrentamento da violência intrafamiliar contra } \\
\text { pessoas idosas pelos profissionais de saúde }{ }^{(28)}\end{array}$ & 2015 & $\begin{array}{l}\text { Violência } \\
\text { intrafamiliar }\end{array}$ & Atenção Primária & Qualitativa & Brasil & LILACS & Artigo \\
\hline $\begin{array}{l}\text { An international collaborative study comparing } \\
\text { Swedish and Japanese } \\
\text { nurses' reactions to elder abuse }{ }^{(29)}\end{array}$ & 2012 & Violência no geral & Atenção Primária & Qualitativa & Suíça e Japão & PsycINF0 & Artigo \\
\hline $\begin{array}{l}\text { Home care nurses' experiences with and } \\
\text { perceptions of elder self-neglect }{ }^{(30)}\end{array}$ & 2015 & Autonegligência & Hospitalar & Qualitativa & $E^{\prime} A^{*}$ & PubMed & Artigo \\
\hline $\begin{array}{l}\text { Elder abuse in Canada and Australia: implications } \\
\text { for nurses }{ }^{(31)}\end{array}$ & 1996 & Violência no geral & $\begin{array}{l}\text { Vários (não especifica } \\
\text { quais) }\end{array}$ & Quantitativa & Canadá e Austrália & Web of Science & Artigo \\
\hline
\end{tabular}

EUA = Estados Unidos da América* Violência no geral = Qualquer violência não especificada no estudo* 


\section{Cuidado de enfermagem forense}

$\mathrm{O}$ cuidado à pessoa idosa em situação de violência realizado por enfermeiros em todo o mundo varia entre os continentes e foi separado por categoria para melhor compreensão e encontra-se descrito no quadro 2.

Quadro 2. Cuidado à pessoa idosa em situação de violência realizado por enfermeiros conforme relato dos estudos

\begin{tabular}{|c|c|}
\hline $\begin{array}{l}\text { Medidas de } \\
\text { prevenção da } \\
\text { violência contra } \\
\text { o idoso }\end{array}$ & 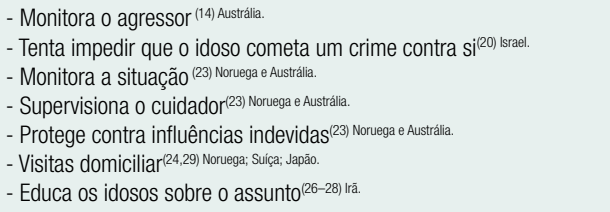 \\
\hline $\begin{array}{l}\text { Recursos } \\
\text { utilizados para } \\
\text { identificação dos } \\
\text { casos de violência } \\
\text { contra o idoso }\end{array}$ & 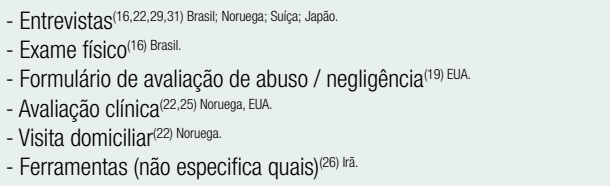 \\
\hline $\begin{array}{l}\text { Intervenções } \\
\text { nas situações de } \\
\text { violência contra } \\
\text { o idoso }\end{array}$ & 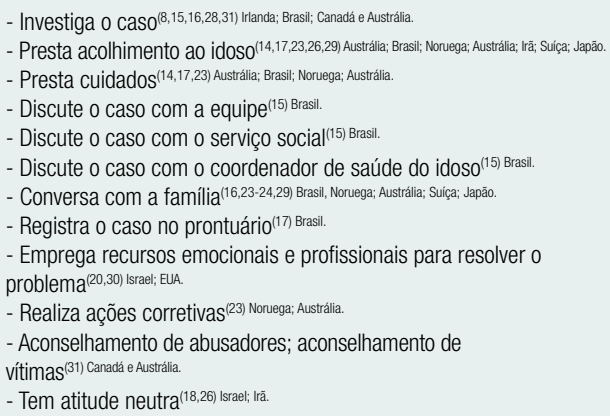 \\
\hline $\begin{array}{l}\text { Encaminhamento } \\
\text { dos casos de } \\
\text { violência contra } \\
\text { o idoso }\end{array}$ & 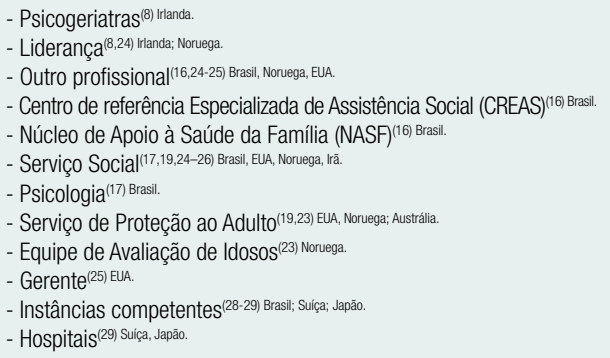 \\
\hline $\begin{array}{l}\text { Denúncia e } \\
\text { notificação dos } \\
\text { casos de violência } \\
\text { contra o idoso }\end{array}$ & $\begin{array}{l}\text { - Aos órgãos competentes }{ }^{(15,19)} \text { Brasil, EUA. } \\
\text { - Ao Conselho do Idoso, Secretaria de Direitos Humanos }{ }^{(17) \text { Brasil. }} \\
\text { - Notifica no Sistema de Informação de Agravos de Notificação } \\
\text { (SINAN)(17) Brasil. } \\
\text { - Polícia }\end{array}$ \\
\hline
\end{tabular}

\section{Dificuldades relatadas pelos enfermeiros para prestar 0 cuidado}

$\mathrm{Na}$ maioria dos estudos elegíveis para esta revisão, $(\mathrm{n}=18 ; 90 \%)$, os enfermeiros relataram dificuldades para prestar o cuidado ao idoso em situaçáo de violência (Quadro 3).
Quadro 3. Dificuldades relatadas pelos enfermeiros ao prestar 0 cuidado ao idoso em situação de violência conforme descrito nos estudos

\begin{tabular}{|c|c|}
\hline $\begin{array}{l}\text { Problemas } \\
\text { organizacionais }\end{array}$ & $\begin{array}{l}\text { - Não ter legislação forte(8) } \\
\text { - Falta de apoio organizacional(14) } \\
\text { - Falta de cooperação profissional e responsabilidade }{ }^{(15)} \\
\text { - Ausência de diretriz }{ }^{(24)} \\
\text { - Fragilidade da integração de serviços públicos voltados ao abuso do } \\
\text { idoso }^{(27)} \\
\text { - Escassez de serviços de saúde mental|(30) } \\
\text { - Barreiras de leis e políticas }{ }^{(30)} \\
\text { - Incapacidade do Serviço de Proteção ao } \text { Adulto }^{(30)} \\
\text {-Dilemas inerentes a prática }\end{array}$ \\
\hline $\begin{array}{l}\text { Capacitação } \\
\text { profissional }\end{array}$ & $\begin{array}{l}\text { - Falta de orientação clara(i) } \\
\text { - Falta de preparo especializado }{ }^{(8,15,26-27)} \\
\text { - Não saber conduziri(15,19,23) } \\
\text { - Dificuldade com os trâmites de notificação } 0^{(16)} \\
\text { - Dificuldade para detecção e intervenção } 0^{(16,18,24)} \\
\text { - Desconhecimento das leis } \\
\text { - Falta de provas }{ }^{(19)}\end{array}$ \\
\hline $\begin{array}{l}\text { Questões } \\
\text { pessoais }\end{array}$ & $\begin{array}{l}\text { - Intimidações / ameaças / represálias }{ }^{(14,28)} \\
\text { - Fatores intrínsecos ao idoso } 0^{(17,22,25,28)} \\
\text { - Não querer se envolver }{ }^{(18,21)} \\
\text { - Insegurança }{ }^{(18,21)} \\
\text { - } 0 \text { impacto dos valores pessoais e experiência }{ }^{(20,25)} \\
\text { - Resistência dos sujeitos envolvidos } \\
\text { - Medo de se expor }\end{array}$ \\
\hline
\end{tabular}

\section{Discussão}

Entre os estudos selecionados para esta revisão, alguns expuseram as medidas de prevenção da VCPI relatadas pelos enfermeiros que participaram das respectivas pesquisas. Na Austrália, os enfermeiros monitoram o agressor para prevenir que novos casos de VCPI aconteçam. ${ }^{(14)} \mathrm{Na}$ Noruega e Austrália, os enfermeiros acompanham o idoso para prevenir que ocorra a VCPI. ${ }^{(23)}$ Além disso, na Noruega e Austrália, os enfermeiros supervisionam o cuidador no seu dia-a-dia, e quando não há melhora no processo do cuidar, sugerem que o cuidador seja afastado da funçáo e solicita cuidados residências para o idoso. Além disso, os enfermeiros protegem o idoso contra influências que o influenciem a impedir sair do ciclo da violência e elaboram planos de segurança para prevenir abusos. ${ }^{(23)}$

Uma revisão sistemática sobre os fatores associados à VCPI, demonstrou que, quando o idoso é dependente para as atividades de vida diária; e instrumentais, o cuidador se sobrecarrega, e, consequentemente aumenta a possibilidade do idoso sofrer violência geral e financeira. ${ }^{(32)}$

$\mathrm{Na}$ Noruega, as visitas diárias ao cliente é uma estratégia utilizada para reduzir o estresse e a carga de trabalho do cuidador ou reduzir a dependência do cliente mais velho do agressor. Além 
disso, eles transferem a vítima para Instituiçóes de Longa Permanência (ILPIs), quando necessário. Enfermeiros da Suíça e Japão também realizam visitas domiciliares para avaliar o ambiente doméstico como medida de prevenção da violência. Essas estratégias são relevantes ao se considerar o número de casos de violência doméstica contra o idoso. Estudo realizado no município de Recife, Pernambuco, Brasil, com o objetivo de investigar a prevalência de violência doméstica contra idosos, demonstra que $78,7 \%$ dos idosos, dentre os 169 investigados, relatam ter sofrido algum tipo de violência, sendo prevalente a negligência, seguido da violência psicológica e financeira. ${ }^{(33)}$

Muitos idosos necessitam de cuidados de terceiros, e caso sofram algum tipo de violência em domicílio, a visita pode ser a única oportunidade de prevenir ou identificar situaçóes de violência. Uma vez que, a vulnerabilidade aumenta com a sobrecarga e despreparo dos familiares e dos cuidadores. ${ }^{(15)}$

No Irã e no Brasil, os enfermeiros lançam mão de ações educativas para a instruçáo de idosos e familiares no intuito de prevenir e combater os casos de violência praticados contra os idosos como evidenciado nos resultados desta scoping review. Essas ações e as supracitadas são consideradas práticas da enfermagem forense conforme descrito na Resoluçáo COFEN 0556/2017.. (34)

Quando se trata dos recursos para identificar os casos de VCPI, os enfermeiros da Noruega lançam mão de técnicas aplicadas pela enfermagem forense, como: entrevista, avaliação clínica e visita domiciliar. ${ }^{(22)} \mathrm{Na}$ Suíça, Japão, Canadá e Austrália, a entrevista também é um recurso utilizado pelos enfermeiros. ${ }^{(29,31)}$ A anamnese, e o exame físico também é uma estratégia aplicada pelos enfermeiros do Brasil. ${ }^{(16)}$ Nos EUA, é utilizado formulário de avaliação da violência e investigação clínica. ${ }^{(19,25)}$

A observação do comportamento do idoso, a comunicação postural, expressão facial, aliado a uma escuta qualificada, pode permitir a identificação de situaçôes vulneráveis, e a partir disso, ser possível elaborar estratégias adequadas para enfrentar a VCPI. ${ }^{(6)}$

Todos estes recursos são ferramentas primordiais para identificar situaçôes de violência, todavia, tem suas particularidades a depender da singularidade de cada caso. Pode-se citar como exemplo, a entrevista (parte do processo de enfermagem), pode não ser aplicada em um idoso que não se comunica, por outro lado, o exame físico, pode ser eficaz para identificar sinais sugestivos de violência. Quando se trata de violência física, o exame físico pode ser a melhor estratégia para a identificação, principalmente quando há hematomas e traumas evidentes. Nesse sentido, muitos idosos buscam a urgência com sinais de queimaduras, fraturas, e outros danos físicos. ${ }^{(35)}$ Sendo importante realizar uma anamnese de qualidade para identificar as causas dos ferimentos

No que se refere as intervençóes realizadas pelos enfermeiros, no Brasil, estas intervençôes são direcionadas a investigação dos casos, assim como no Canadá e Austrália. O acolhimento das vítimas de violência e diálogo com a família do agressor são realizados pelos enfermeiros do Brasil, Austrália, Noruega, Irã, Suíça e Japão. (14,17,23,26,29) $^{2}$

No Brasil, o enfermeiro discute o caso de VCPI com a equipe, com o serviço social e coordenador de saúde do idoso, e também, registra o caso no prontuário. Nos EUA, os recursos emocionais e profissionais são uma estratégia adotada pelos enfermeiros para tentar resolver o caso. Medidas corretivas são utilizadas por enfermeiros na Austrália e Noruega, mas os estudos não as especificam. Nos estudos; realizados em Israel e Irã, respectivamente, revelam atitudes neutras dos enfermeiros em relação a intervir nos casos de VCPI. ${ }^{(16,18)} \mathrm{O}$ que caracteriza uma abordagem náo condizente com a função do enfermeiro enquanto atuante do processo saúde e doença. No Brasil, caso isso ocorra, o enfermeiro pode ser punido na forma da lei, por ser "dever de todos prevenir a ameaça ou violação aos direitos do idoso" conforme lei no $10.741 / 2003$. ${ }^{(36)}$

Nesse sentido, identificar situaçóes de vulnerabilidade em que o idoso se encontra é uma tarefa complexa, e por isso, deve-se considerar uma abordagem holística e singular. Um estudo realizado no município do Recife-PE, com 169 idosos, revelou que, a negligência contra o idoso foi o tipo de violência mais prevalente, representando $58,5 \%$ dos casos, seguida da violência financeira $21,5 \%$ e psicológica $14,0 \% .^{(33)}$ Todas estas, podendo ocorrer no 
ambiente familiar, o que dificulta a identificação e prevenção dos casos. Desse modo, a visita domiciliar pode ser um recurso aplicável para auxiliar na identificação de casos de VCPI.

No que tange o encaminhamento dos casos de VCPI, os enfermeiros encaminham para os diferentes profissionais que componham a equipe multidisciplinar e, quando necessário, direcionam a hospitais e instâncias competentes. Encaminhar para o serviço social foi relatado em cinco estudos, realizados no Brasil, EUA, Noruega, Irã. ${ }^{(17,19,23-26)}$

$\mathrm{O}$ encaminhamento dos casos de violência para outras instâncias é elementar para dar continuidade a resolução do caso. No entanto, um estudo realizado no município de Goiânia-GO, com objetivo de analisar o perfil da pessoa idosa vítima de violência atendida em um hospital de urgência, no período de um ano, revelou que não havia articulação e comunicação entre as instituiçóes especializadas para onde os idosos foram encaminhados, desse modo, interrompendo a assistência. ${ }^{(37)}$

No tocante as denúncias e notificaçóes dos casos de VCPI, no Brasil, os enfermeiros denunciam as instâncias competentes, como Conselho do idoso, Secretaria de Direitos Humanos e polícia. Além disso, notificam no SINAN. A denúncia aos órgãos competentes foi relatada em um único estudo realizado nos EUA, ${ }^{(19)}$, a denúncia especificamente a polícia também foi relatada nos estudos realizados na Noruega, Austrália e Irã. ${ }^{(23,24,26)}$

Nos últimos anos, o Brasil tem investido em políticas que visam combater a violência. Em 2001, o Ministério da Saúde instituiu a Política Nacional de Redução a Morbimortalidade por Acidentes e Violências, além disso, foi publicado um manual instrutivo orientando o preenchimento da ficha de notificação dos casos de violência. ${ }^{(38)}$ Em 2011, com a Lei no 12.461 , a notificação compulsória dos casos de VCPI, passou a ser obrigatória por todos os profissionais de saúde que trabalham em serviços públicos ou privados no Brasil. ${ }^{(39)}$ Ademais, essa mesma lei traz a obrigatoriedade da comunicação dos casos de VCPI, “a quaisquer dos seguintes órgãos: autoridade policial, Ministério Público, Conselho Municipal do Idoso, Conselho Estadual do Idoso, e/ou Conselho Nacional do Idoso".
Os enfermeiros relatam as dificuldades diante da atuação em situaçôes de VCPI, sejam pessoais, profissionais e até mesmo de infraestrutura para prevenir casos e/ou atender as vítimas perpetradas. E, soma-se a isso, a ausência de programas educacionais nos serviços para melhorar o desempenho dos enfermeiros em diferentes níveis de prevenção de VCPI. ${ }^{(26,27)}$

Os enfermeiros, ficam inseguros ao manejar situações de VCPI por falta de orientação clara sobre como definir e gerenciar o abuso dentro das relaçôes de confiança, e por sentir fragilidade na legislação. ${ }^{(8)}$ Além disso, a falta de apoio da secretaria de saúde e outros departamentos municipais, a ausência de cooperação profissional, responsabilidade, falta de treinamento, ausência de capacidade para agir em alguns casos, os trâmites de notificação, detecção e intervenção são obstáculos que impactam o enfrentamento da VCPI. ${ }^{(15)}$

Do mesmo modo, os diversos fatores intrínsecos ao idoso contribuem para dificultar a identificação da violência pelos enfermeiros, como medo, vergonha e omissão por medo do agressor ou por não querer denunciá-los por questóes de afinidades parentais. ${ }^{(17)} \mathrm{E}$ assim surge o dilema que se refere ao direito dos idosos de escolher como viver suas vidas versus o desejo e obrigação do enfermeiro de ajudar e desempenhar suas funções profissionais. ${ }^{(20)}$

Destaca-se que as açóes dos enfermeiros generalistas, evidenciadas no presente estudo vão de encontro com às práticas da enfermagem forense, preconizadas pela Resolução COFEN no 556/2017, que envolvem: denunciar os casos de violência a autoridades, planejar entrevistas, a necessidade de envolver outros profissionais e familiares, identificar vestígios de relevância criminal, identificar indicadores de suspeita de violência, documentar todas as informaçôes relevantes, coordenar a transição da vítima entre os cenários de cuidados, orientar o acesso a recursos jurídicos, envolver outros profissionais para garantir a continuidade do cuidado, utilizar técnicas de entrevistas, observar comportamentos da vítima e demais envolvidos, dentre outros. ${ }^{(34)}$

Os desafios ao lidar com situaçóes de violência são inúmeros, por isso a expansão da EF deve ser incentivada em todo o mundo. Por permitir uma as- 
sistência completa às vítimas, perpetradores e familiares, utilizando ferramentas de rastreamento como supracitado para detecção precoce e implementação de cuidados sistematizados de enfermagem, ${ }^{(2)}$ permitindo manter um registro de todas as informaçôes, pois elas podem ser consideradas como prova em processos judiciais e respaldo legal do profissional de saúde.

Ressalta-se como limitação do estudo, a ausência de publicaçóes envolvendo especificamente o enfermeiro especialista em enfermagem forense.

\section{Conclusão}

Os enfermeiros lançam mão de estratégias diversificadas dependendo do contexto da violência e do país que atuam. É possível perceber o cuidado forense nas açóes dos enfermeiros generalistas, ainda que não necessariamente detenham o conhecimento de que estão utilizando estratégias que fazem parte da especialidade. Os estudos revelam que os enfermeiros apoiam os idosos vítima de violência, investigam os casos através de avaliação clínica, denunciam às autoridades e acionam a equipe multiprofissional na tentativa de solucionar o problema. No entanto, estes profissionais encontram barreiras para identificar e manejar o idoso em situação de violência, uma vez, que se trata de um fenômeno complexo de difícil detecção. Entre estas barreiras, estão a falta de apoio da gestão, a incapacidade para identificar as situaçóes de violência, a falta de treinamentos e, a ausência de políticas públicas. Nesse sentido, os resultados encontrados poderão dar subsídios para se planejar a ascensão da enfermagem forense, e qualificação dos profissionais generalistas, a partir deste conhecimento. Consequentemente, promover assistência eficiente a essas vítimas, melhorando sua qualidade de vida.

\section{Agradecimentos}

O presente trabalho foi realizado com o apoio do Conselho Nacional de Desenvolvimento Científico e Tecnológico - CNPq através da Chamada
Universal MCTIC/CNPq 2018 e, da Coordenação de Aperfeiçoamento de Pessoal de Nível Superior Brasil (CAPES) - Código de Financiamento 001.

\section{Referências}

1. Felipe HR, Cunha M, Ribeiro VS, Zamarioli CM, Santos CB, Duarte JC, et al. Questionário de Conhecimentos sobre Práticas de Enfermagem Forenses: adaptação para o Brasil e a propriedades psicométricas. Rev Enferm Ref. 2019;4(23):99-110.

2. International Association of Forensic Nurses (IAFN). History of the Association. Elkridge: IAFN; 2018 [cited 2021 Feb 21]. Available from: https://www.forensicnurses.org/page/AboutUS

3. Pereira de Paiva MH, Pinheiro Lages L, Cavalcanti de Medeiros Z. Studies on forensic nursing in Brazil: a systematic review of the literature. Int Nurs Rev. 2017;64(2):286-95. Review.

4. Santos AA, Silva JF, Ferreira MB, Conceição VL, Alves DM. Estado da arte da Enfermagem Forense no cenário atual da saúde. Rev Eletrônica Acervo Saúde. 2019;27:e1015.

5. Associação Brasileira de Enfermagem Forense (ABEFORENSE). Cartilha de Orientação de Enfermagem Forense. Violência, identifique, notifique, denuncie. Aracaju: ABEFORENSE; 2017. 56 p.

6. Castro VC, Rissardo LK, Carreira L. Violence against the Brazilian elderlies: an analysis of hospitalizations. Rev Bras Enferm. 2018;71(Suppl 2):777-85.

7. Yon Y, Mikton CR, Gassoumis ZD, Wilber KH. Elder abuse prevalence in community settings: a systematic review and meta-analysis. Lancet Glob Health. 2017;5(2):e147-56. Review.

8. Phelan A, McCarthy S, McKee J. Safeguarding staff's experience of cases of financial abuse. Br J Soc Work. 2018;48(4):924-42.

9. Garbin CA, Dias IA, Rovida TA, Garbin AJ. Desafıos do profıssional de saúde na notifıcação da violência: obrigatoriedade, efetivação e encaminhamento. Ciên Saúde Coletiva. 2015;20(6):1879-90.

10. Camacho AC, Alves RR. Mistreatment against the elderly in the nursing perspective: an integrative review. J Nurs UFPE Online. 2015;9(2):92735.

11. Santos FD, de Lima Saintrain MV, de Souza Vieira LJ, Gomes Marques Sampaio E. Characterization and prevalence of elder abuse in Brazil. J Interpers Violence. 2018 Jun 1:886260518781806.

12. Tricco AC, Lillie E, Zarin W, O'Brien KK, Colquhoun H, Levac D, et al. PRISMA Extension for Scoping Reviews (PRISMA-ScR): Checklist and Explanation. Ann Intern Med. 2018;169(7):467-73.

13. Peters MD, Godfrey CM, Khalil H, Mclnerney P, Parker D, Soares CB. Guidance for conducting systematic scoping reviews. Int J Evid Based Healthc. 2015;13(3):141-6.

14. Cairns J, Vreugdenhil A. Working at the frontline in cases of elder abuse: 'it keeps me awake at night'. Australas J Ageing. 2014;33(1):59-62.

15. Oliveira KS, Carvalho FP, Oliveira LC, Simpson CA, Silva FT, Martins AG. Violência contra idosos: concepções dos profissionais de enfermagem acerca da detecção e prevenção. Rev Gaúcha Enferm. 2018;39:e57462.

16. Musse J0, Rios MH. Atuação do enfermeiro perante a violência doméstica sofrida pelo idoso. Estud Interdiscip Envelhec. 2015;20(2):365-79. 
17. Azevedo CO, Silva TA. Cuidados de enfermagem para detecção de violência contra idosos. Rev Pró-UniverSUS. 2019;10(1):55-9.

18. Almogue A, Weiss A, Marcus EL, Beloosesky Y. Attitudes and knowledge of medical and nursing staff toward elder abuse. Arch Gerontol Geriatr. 2010;51(1):86-91.

19. Miller CA. Elder Abuse. The nurse's perspective. Clin Gerontol. 2005;28(1-2):105-33.

20. Band-Winterstein T. Nurses' encounters with older adults engaged in self-neglectful behaviors in the community: a qualitative study. J Appl Gerontol. 2018;37(8):965-89.

21. Ko C, Koh CK. Factors related to korean nurses' Willingness to report suspected elder abuse. Asian Nurs Res (Korean Soc Nurs Sci). 2012;6(3):115-9.

22. Sandmoe A, Kirkevold M. Nurses' clinical assessments of older clients who are suspected victims of abuse: an exploratory study in community care in Norway. J Clin Nurs. 2011;20(1-2):94-102.

23. Sandmoe A, Kirkevold M, Ballantyne A. Challenges in handling elder abuse in community care. An exploratory study among nurses and care coordinators in Norway and Australia. J Clin Nurs. 2011;20(23-24):3351-63.

24. Sandmoe A, Kirkevold M. Identifying and handling abused older clients in community care: the perspectives of nurse managers. Int J Older People Nurs. 2013;8(2):83-92.

25. Daly JM, Schmeidel Klein AN, Jogerst GJ. Critical care nurses' perspectives on elder abuse. Nurs Crit Care. 2012;17(4):172-9.

26. Alipour A, Fotokian Z, Shamsalinia A, Ghaffari F, Hajiahmadi M. The relationship between nurses' recognition regarding elder abuse and their attitudes and performance in dealing with elder abuse induced by iranian family caregivers. Open Nurs J. 2019;13(1):116-22.

27. Almeida CA, Silva Neto MC, Carvalho FM, Lago EC. Aspectos relacionados à violência contra 0 idoso: concepção do enfermeiro da estratégia saúde da família. J Res Fundam Care Online. 2019;11(Esp):404-10.

28. Rocha EN, Vilela AB, Silva DM. Enfrentamento da violência intrafamiliar contra pessoas idosas pelos profissionais de saúde. Kairós Gerontol. 2015;18(4):29-46.

29. Erlingsson C, Ono M, Sasaki A, Saveman BI. An international collaborative study comparing Swedish and Japanese nurses' reactions to elder abuse. J Adv Nurs. 2012;68(1):56-68.
30. Johnson YO. Home care nurses' experiences with and perceptions of elder self-neglect. Home Healthc Now. 2015;33(1):31-7.

31. Trevitt C, Gallagher E. Elder abuse in Canada and Australia: implications for nurses. Int J Nurs Stud. 1996;33(6):651-9.

32. Santos MA, Moreira RS, Faccio PF, Gomes GC, Silva VL. Factors associated with elder abuse: a systematic review of the literature. Ciên Saúde Coletiva. 2020;25(6):2153-75.

33. Barros RL, Leal MC, Marques AP, Lins ME. Domestic violence against elderly people assisted in primary care. Saúde Debate. 2019;43(122):793-804.

34. Conselho Federal de Enfermagem (COFEN). Resolução COFEN $n^{\circ}$. 556/2017. Brasília (DF): COFEN; 2017 [citado 2021 Fev 21]. Disponível em: http://www.cofen.gov.br/resolucao-cofen-no-05562017_54582. html

35. Pillemer K, Burnes D, Riffin C, Lachs MS. Elder Abuse: Global Situation, Risk Factors, and Prevention Strategies. Gerontologist. 2016;(Suppl 2):S194-205. Review.

36. Brasil. Presidência da República. Casa Civil. Subchefia para Assuntos Jurídicos. Lei $n^{0} 10.741$, de $1^{\circ}$ de outubro de 2003. Dispõe sobre 0 Estatuto do Idoso e dá outras providências. Brasília (DF): Presidência da República; 2003. Disponível em: http://www.planalto.gov.br/ccivil_03/ leis/2003/110.741.htm

37. Soares MC, Barbosa AM. Perfil de idosos vítimas de violência atendidos em um hospital de urgências. Rev Cient Esc Saúde Pública Goiás "Candido Santiago". 2020;6(1):18-34.

38. Brasil. Ministério da Saúde. Secretaria de Vigilância em Saúde. Departamento de Vigilância de Doenças e Agravos Não Transmissíveis e Promoção da Saúde. Viva: instrutiva notificação de violência interpessoal e autoprovocada. 2a ed. Brasília (DF): Ministério da Saúde; 2016. Disponível em: http://bvsms.saude. gov.br/bvs/publicacoes/viva_instrutivo_violencia_interpessoal_ autoprovocada_2ed.pdf

39. Brasil. Câmara dos Deputados. Lei n 12.461, de 26 de julho de 2011. Altera a Lei $n^{0} 10.741$, de $1^{\circ}$ de outubro de 2003, para estabelecer a notificação compulsória dos atos de violência praticados contra o idoso atendido em serviço de saúde. Brasília (DF): Câmara dos Deputados; 2011. Disponível em: https://www2.camara.leg.br/legin/fed/lei/2011/ lei-12461-26-julho-2011-611103-norma-pl.html 